\title{
On Absolute Continuity of Conjugations between Circle Maps with Break Points
}

\author{
Habibulla Akhadkulovi,2 and Mohd Salmi Md Noorani ${ }^{1}$ \\ ${ }^{1}$ School of Mathematical Sciences, Faculty of Science and Technology, University Kebangsaan Malaysia, \\ Bangi, 43600 Selangor, Malaysia \\ ${ }^{2}$ Faculty of Mathematics and Mechanics, Samarkand State University, Boulevard Street 15, \\ 703004 Samarkand, Uzbekistan
}

Correspondence should be addressed to Habibulla Akhadkulov, akhadkulov@yahoo.com

Received 31 August 2012; Accepted 23 October 2012

Academic Editor: Ivanka Stamova

Copyright (c) 2012 H. Akhadkulov and M. S. Md Noorani. This is an open access article distributed under the Creative Commons Attribution License, which permits unrestricted use, distribution, and reproduction in any medium, provided the original work is properly cited.

Let $T_{1}$ and $T_{2}$ be piecewise smooth circle homeomorphisms with break points and identical irrational rotation numbers. We provide one sufficient and necessary condition for the absolute continuity of conjugation map between $T_{1}$ and $T_{2}$.

\section{Introduction and Statement of Results}

Let $S^{1}=\mathbb{R} / \mathbb{Z}$ with clearly defined orientation, metric, Lebesgue measure, and the operation of addition be the unit circle. Let $\pi: \mathbb{R} \rightarrow S^{1}$ denote the corresponding projection mapping that "winds" a straight line on the circle. An arbitrary homeomorphism $T$ that preserves the orientation of the unit circle $S^{1}$ can "be lifted" on the straight line $\mathbb{R}$ in the form of the homeomorphism $L_{T}: \mathbb{R} \rightarrow \mathbb{R}$ with property $L_{T}(x+1)=L_{T}(x)+1$ that is connected with $T$ by relation $\pi \circ L_{T}=T \circ \pi$. This homeomorphism $L_{T}$ is called the lift of the homeomorphism $T$ and is defined up to an integer term. The most important arithmetic characteristic of the homeomorphism $T$ of the unit circle $S^{1}$ is the rotation number

$$
\rho(T)=\lim _{i \rightarrow \infty} \frac{L_{T}^{i}(x)}{i} \bmod 1
$$

where $L_{T}$ is the lift of $T$ with $S^{1}$ to $\mathbb{R}$. Here and below, for a given map $F, F^{i}$ denotes its $i$ th iteration. Poincaré proved that the above limit exists, does not depend on the initial point 
$x \in \mathbb{R}$ of the lifted trajectory, and, up to additional of an integer, does not depend on the lift $L_{T}$ (see [1]). The rotation number $\rho=\rho(T)$ is irrational if and only if the homeomorphism $T$ has no periodic point. Hereafter, we will always assume that $\rho$ is irrational and use its decomposition in an infinite continued fraction (see [2])

$$
\rho=\frac{1}{k_{1}+\frac{1}{k_{2}+\frac{1}{\frac{\cdots}{k_{n}+\frac{1}{\cdots}}}}}:=\left[k_{1}, k_{2}, \ldots, k_{n}, \ldots\right] .
$$

The value of a "countable-floor" fraction is the limit of the sequence of rational convergents $p_{n} / q_{n}=\left[k_{1}, k_{2}, \ldots, k_{n}\right]$. The positive integers $k_{n}, n \geq 1$, called incomplete multiples, are defined uniquely for irrational $\rho$. The mutually prime positive integers $p_{n}$ and $q_{n}$ satisfy the recurrent relations $p_{n}=k_{n} p_{n-1}+p_{n-2}$ and $q_{n}=k_{n} q_{n-1}+q_{n-2}$ for $n \geq 1$, where it is convenient to define $p_{-1}=0, q_{-1}=1$ and $p_{0}=1, q_{0}=k_{1}$. Given a circle homeomorphism $T$ with irrational rotation number $\rho$, one may consider a marked trajectory (i.e., the trajectory of a marked point) $\xi_{i}=T^{i} \xi_{0} \in S^{1}$, where $i \geq 0$, and pick out of it the sequence of the dynamical convergents $\xi_{q_{n}}, n \geq 0$, indexed by the denominators of consecutive rational convergents to $\rho$. We will also conventionally use $\xi_{q_{-1}}=\xi_{0}-1$. The well-understood arithmetical properties of rational convergents and the combinatorial equivalence between $T$ and rigid rotation $R_{\rho}: \xi \rightarrow \xi+\rho$ mod 1 imply that the dynamical convergents approach the marked point, alternating their order in the following way:

$$
\xi_{q_{-1}}<\xi_{q_{1}}<\xi_{q_{3}}<\cdots<\xi_{q_{2 m+1}}<\cdots<\xi_{0}<\cdots<\xi_{q_{2 m}}<\cdots<\xi_{q_{2}}<\xi_{q_{0}}
$$

We define the $n$th fundamental interval $\Delta^{n}\left(\xi_{0}\right)$ as the circle arc $\left[\xi_{0}, T^{q_{n}}\left(\xi_{0}\right)\right]$ for even $n$ and as $\left[T^{q_{n}}\left(\xi_{0}\right), \xi_{0}\right]$ for odd $n$. For the marked trajectory, we use the notation $\Delta_{0}^{n}=\Delta^{n}\left(\xi_{0}\right), \Delta_{i}^{n}=$ $\Delta^{n}\left(\xi_{i}\right)=T^{i} \Delta_{0}^{n}$. It is well known that the set $\mathbf{P}_{n}\left(\xi_{0}, T\right)=\mathbf{P}_{n}(T)$ of intervals with mutually disjoint interiors defined as

$$
\mathbf{P}_{n}(T)=\left\{\Delta_{i}^{n-1}, 0 \leq i<q_{n} ; \Delta_{j}^{n}, 0 \leq j<q_{n-1}\right\}
$$

determines a partition of the circle for any $n$. The partition $\mathbf{P}_{n}(T)$ is called the $n$th dynamical partition of the point $\xi_{0}$. Obviously the partition $\mathbf{P}_{n+1}(T)$ is a refinement of the partition $\mathbf{P}_{n}(T)$ : indeed the intervals of order $n$ are members of $\mathbf{P}_{n+1}(T)$ and each interval $\Delta_{i}^{n-1} \in \mathbf{P}_{n}(T) 0 \leq i<$ $q_{n}$, is partitioned into $k_{n+1}+1$ intervals belonging to $\mathbf{P}_{n+1}(T)$ such that

$$
\Delta_{i}^{n-1}=\Delta_{i}^{n+1} \cup \bigcup_{s=0}^{k_{n+1}-1} \Delta_{i+q_{n-1}+s q_{n}}^{n}
$$

Class $\mathfrak{B}$-homeomorphisms. These are orientation-preserving circle homeomorphisms $T$ differentiable except in finite number break points at which left and right derivatives, denoted, respectively by $D T_{-}$and $D T_{+}$, exist, and such that 
(i) there exist constants $0<c_{1}<c_{2}<\infty$ with $c_{1}<D T(x)<c_{2}$ for all $x \in S^{1} \backslash \mathrm{BP}(T)$, $c_{1}<D T_{-}\left(x_{b}\right)<c_{2}$ and $c_{1}<D T_{+}\left(x_{b}\right)<c_{2}$ for all $x_{b} \in \mathrm{BP}(T)$, with $\mathrm{BP}(T)$ the set of break points of $T$ on $S^{1}$;

(ii) DT has bounded variation.

The ratio $\sigma_{T}(c):=\left(D T_{-}(c)\right) /\left(D T_{+}(c)\right)$ is called the jump of $T$ in $c$ or the $T$-jump. General $\mathfrak{B}$-homeomorphisms with one break point was first studied by Khanin and Vul in [3]. Among other results it was proved by these authors that their renormalizations approximate fractional linear transformations. Let $T$ be an orientation preserving $C^{1}$-diffeomorphism of the circle. If the rotation number $\rho$ is irrational and $D T$ is of bounded variation then, by a well-known theorem of Denjoy, $T$ is conjugate to the rigid rotation $R_{\rho}$ (see [1]). The conjugation means that there exists an essentially unique homeomorphism $h$ of the circle such that $T=h^{-1} \circ R_{\rho} \circ h$. In this context, a natural question to ask is under what condition the conjugacy is smooth? Several authors, for example [4-6] have shown that if $T$ is $C^{2+\alpha}, \alpha>0$ and $\rho$ satisfies certain diophantine condition then the conjugacy will be at least $C^{1}$.

The classical result of Denjoy can be easily extended to the case of $\mathfrak{B}$-homeomorphisms. Next we consider the problem of the regularity of the conjugating map between two class $\mathfrak{B}$-homeomorphisms with one break point and coinciding irrational rotation numbers. The case of one break point with the same jump ratios, so called rigidity problem, was studied in detail by Teplinskii and Khanin in [7]. Let $\rho=\left[k_{1}, k_{2}, \ldots, k_{n}, \ldots\right]$ be the continued fraction expansion of the irrational rotation number $\rho$ and define

$$
M_{o}=\left\{\rho: \exists C>0, \forall n \in \mathbb{N}, k_{2 n-1} \leq C\right\}, \quad M_{e}=\left\{\rho: \exists C>0, \forall n \in \mathbb{N}, k_{2 n} \leq C\right\} .
$$

The main result of [7] is as follows.

Theorem 1.1. Let $T_{i} \in C^{2+\alpha}\left(S^{1} \backslash\left\{b_{i}\right\}\right), i=1,2, \alpha>0$ be $\mathfrak{B}$-homeomorphisms with one break point that have the same jump ratio $\sigma$ and the same irrational rotation number $\rho \in(0,1)$. In addition, let one of the following restrictions be true: either $\sigma>1$ and $\rho \in M_{e}$ or $\sigma<1$ and $\rho \in M_{o}$. Then the map $h$ conjugating the homeomorphisms $T_{1}$ and $T_{2}$ is a $C^{1}$-diffeomorphism.

In the case of different jump ratios, the following theorem was proved in [8] by Dzhalilov et al.

Theorem 1.2. Let $T_{i} \in C^{2+\alpha}\left(S^{1} \backslash\left\{b_{i}\right\}\right), i=1,2, \alpha>0$ be $\mathfrak{B}$-homeomorphisms with one break point that have different jump ratio and the same irrational rotation number $\rho \in(0,1)$. Then the map $h$ conjugating the homeomorphisms $T_{1}$ and $T_{2}$ is a singular function, that is, is continuous on $S^{1}$ and $\operatorname{Dh}(x)=0$ a.e. with respect to Lebesgue measure.

Let $T_{1}$ and $T_{2}$ be $\mathfrak{B}$-homeomorphisms with identical irrational rotation number $\rho$. Now, we consider dynamical partitions $\mathbf{P}_{n}\left(\xi, T_{1}\right)=\mathbf{P}_{n}\left(T_{1}\right)$ and $\mathbf{P}_{n}\left(h(\xi), T_{2}\right)=\mathbf{P}_{n}\left(T_{2}\right)$ appropriate to the homeomorphisms $T_{1}$ and $T_{2}$. Denote by $\widehat{\Delta}^{n}$ intervals of partition of $\mathbf{P}_{n}\left(T_{2}\right)$. Since the function $h$ is a conjugation function between $T_{1}$ and $T_{2}$, so we have $h\left(\Delta^{n}\right)=\widehat{\Delta}^{n}$ for any $\Delta^{n} \in \mathbf{P}_{n}\left(T_{1}\right)$. Denote by $|A|$ the Lebesgue measure of the corresponding set of $A \subset S^{1}$. Our purpose in this paper is to give some criteria for the absolute continuity of the conjugation map $h$. Our first main result is the following. 
Theorem 1.3. Assume the rotation number $\rho$ is irrational of bounded type. Suppose that there exist a sequence $\left(\tau_{n}\right)$ such that $\sum_{n=1}^{\infty} \tau_{n}^{2}<\infty$ with

$$
\left|\frac{\left|\Delta_{1}\right|}{\left|\Delta_{2}\right|}-\frac{\left|\widehat{\Delta}_{1}\right|}{\left|\widehat{\Delta}_{2}\right|}\right| \leq \tau_{n}
$$

for each pair of adjacent intervals $\Delta_{1}, \Delta_{2} \in \mathbf{P}_{n}\left(T_{1}\right)$ for all $n>1$. Then the conjugation map $h$ is absolutely continuous function.

In the proof of Theorem 1.3, we will use the consideration of theory of martingales. The idea of using theory of martingales was established in [9] by Katznelson and Ornstein. Our second main result is the following.

Theorem 1.4. Let $T_{1}$ and $T_{2}$ be $\mathfrak{B}$-homeomorphisms with identical irrational rotation number $\rho$. If the conjugation map $h$ is a absolutely continuous function, then for all $\delta>0$, the sequence of Lebesgue measure of the set $\left|\left\{x:\left|\log D T_{2}^{q_{n}}(h(x))-\log D T_{1}^{q_{n}}(x)\right| \geq \delta\right\}\right|$ tends to 0 when $n$ goes to $+\infty$.

\section{The Denjoy Theory and Ergodicity of $\mathfrak{B}$-Homeomorphisms}

The assertions listed below, which are valid for any orientation-preserving homeomorphism $T \in \mathfrak{B}$ with irrational rotation number $\rho$, constitute classical Denjoy theory. Their elementary proofs can be found in $[10,11]$.

(a) Generalized Denjoy estimate; let $\xi_{0} \in S^{1}$ be a continuity point of $D T^{q_{n}}$, then the following inequality holds: $e^{-v} \leq D T^{q_{n}}\left(\xi_{0}\right) \leq e^{v}$, where $v=\operatorname{Var}_{S^{1}} \log D T$.

(b) Exponential refinement; there exists a universal constant $C_{1}=C_{1}(T)$ such that $\left|\Delta_{0}^{n+m}\right| \leq C_{1} \lambda^{m}\left|\Delta_{0}^{n}\right|$, where $\lambda=\left(1+e^{-v}\right)^{-1 / 2}$.

(c) Bounded geometry; let rotation number $\rho$ is bounded type that is the coefficients in continued fraction expansion of $\rho$ are bounded. Then there exist universal constants $C_{2}=C_{2}(T), C_{3}=C_{3}(T)$ such that $0<C_{2}<1$ and $C_{3} \leq 1$ with

(i) each pair of adjacent intervals of $\mathbf{P}_{n}(T)$ are $C_{2}$-comparable that is their ratio of lengths belongs to $\left[C_{2}, C_{2}^{-1}\right]$;

(ii) an interval $\Delta^{n+1}$ of $\mathbf{P}_{n+1}(T)$ is $C_{3}$-comparable to the interval $\Delta^{n}$ of $\mathbf{P}_{n}(T)$ that contains it: $C_{3}\left|\Delta^{n}\right| \leq\left|\Delta^{n+1}\right|$.

(d) Generalized Finzi estimate; suppose $\xi \in S^{1}, \eta \in \Delta^{n-1}(\xi)$ and $\xi, \eta$ are continuity points of $D T^{q_{n}}$. Then for any $0 \leq k<q_{n}$, the following inequality holds: $\left|\log D T^{k}(\xi)-\log D T^{k}(\eta)\right| \leq v$.

Let $\left(S^{1}, \mathfrak{G}, \mu\right)$ be a measure space and $F: S^{1} \rightarrow S^{1}$ be a measurable map.

Definition 2.1. The set $A \in \mathfrak{G}$ is said to be invariant with respect to the measurable $F$, if $A=$ $F^{-1} A$.

Definition 2.2. A measurable map $F: S^{1} \rightarrow S^{1}$ is said to be ergodic with respect measure $\mu$ if the measure $\mu(A)$ of any invariant set $A$ equals 0 or 1 .

Let $\xi_{0} \in S^{1}$, denote by $V_{n}=\Delta^{n}\left(\xi_{0}\right) \cup \Delta^{n-1}\left(\xi_{0}\right)$. 
Lemma 2.3. Let $T$ be a $\mathfrak{B}$-homeomorphism with irrational rotation number $\rho$. Suppose $\xi \in V_{n}$ and $\xi$ be a continuity point of $D T^{q_{n}}$. Then for any $0 \leq k<q_{n}$, the following inequality holds:

$$
e^{-v} \frac{\left|T^{k}\left(V_{n}\right)\right|}{\left|V_{n}\right|} \leq D T^{k}(\xi) \leq e^{v} \frac{\left|T^{k}\left(V_{n}\right)\right|}{\left|V_{n}\right|}
$$

Proof. Let the system of intervals $\supset=\left\{I: I \subset V_{n}\right.$, and the map $D T^{q_{n}}$ is continuous on $\left.I\right\}$ be continuity intervals of $D T^{q_{n}}$. Let $\xi \in \Delta^{n-1}\left(\xi_{0}\right)$. Then, by the mean value theorem, for any $0 \leq k<q_{n}$, we have

$$
\frac{\left|T^{k}\left(\Delta^{n-1}\left(\xi_{0}\right)\right)\right|}{D T^{k}(\xi)\left|\Delta^{n-1}\left(\xi_{0}\right)\right|}=\frac{D T^{k}\left(z_{1}\right)\left|I_{1}\right|+D T^{k}\left(z_{2}\right)\left|I_{2}\right|+\cdots+D T^{k}\left(z_{d}\right)\left|I_{d}\right|}{D T^{k}(\xi)\left|\Delta^{n-1}\left(\xi_{0}\right)\right|}
$$

where $z_{i} \in I_{i} \subset \Delta^{n-1}\left(\xi_{0}\right)$ and $I_{i} \in \mathcal{O}, 1 \leq i \leq d$. If $\xi \in \Delta^{n}\left(\xi_{0}\right)$ then we have

$$
\frac{\left|T^{k}\left(\Delta^{n}\left(\xi_{0}\right)\right)\right|}{D T^{k}(\xi)\left|\Delta^{n}\left(\xi_{0}\right)\right|}=\frac{D T^{k}\left(y_{1}\right)\left|J_{1}\right|+D T^{k}\left(y_{2}\right)\left|J_{2}\right|+\cdots+D T^{k}\left(y_{t}\right)\left|J_{t}\right|}{D T^{k}(\xi)\left|\Delta^{n}\left(\xi_{0}\right)\right|}
$$

where $y_{i} \in J_{i} \subset \Delta^{n}\left(\xi_{0}\right)$ and $J_{i} \in \mathcal{O}, 1 \leq i \leq t$. Apply generalized Finzi estimate to the righthand side of relations (2.2) and (2.3), we get

$$
e^{-v} \leq \frac{\left|T^{k}\left(\Delta^{n-1}\left(\xi_{0}\right)\right)\right|}{D T^{k}(\xi)\left|\Delta^{n-1}\left(\xi_{0}\right)\right|} \leq e^{v}, \quad e^{-v} \leq \frac{\left|T^{k}\left(\Delta^{n}\left(\xi_{0}\right)\right)\right|}{D T^{k}(\xi)\left|\Delta^{n}\left(\xi_{0}\right)\right|} \leq e^{v} .
$$

Finally, we get

$$
e^{-v} \leq \frac{\left|T^{k}\left(V_{n}\right)\right|}{D T^{k}(\xi)\left|V_{n}\right|} \leq e^{v}
$$

Lemma 2.4. Let $T$ be a $\mathfrak{B}$-homeomorphism of the circle $S^{1}$ with irrational rotation number $\rho$, then $T$ is ergodic with respect to Lebesgue measure.

Proof. Suppose that there exist an invariant set $A$ of positive but not full Lebesgue measure $|A|$. Then by the Lebesgue Density Theorem, $A$ has a density point $z$. We fix an arbitrary $\epsilon>0$. By definition of density points, we can find a $\delta>0$ such that for any interval $[a, b]$ satisfying the conditions $z \in[a, b],[a, b] \subset(z-\delta, z+\delta)$, we have $|A \cap[a, b]| \geq(1-\epsilon)|[a, b]|$, or, in other words, $\left|A^{c} \cap[a, b]\right|<\epsilon|[a, b]|$, where $A^{c}$ denotes the complement of $A$. Now, we choose such $n$ that $V_{n}=\Delta^{n}(z) \cup \Delta^{n-1}(z) \subset(z-\delta, z+\delta)$. We can check that $\bigcup_{k=0}^{q_{n}-1} T^{k}\left(V_{n}\right)=S^{1}$ and each 
point of the circle belongs to at most two intervals of this cover. Hence, the set $A^{c}$ is invariant with respect to $T$, using the above lemma, we get

$$
\left|A^{c}\right|=\sum_{k=0}^{q_{n}-1}\left|A^{c} \cap T^{k}\left(V_{n}\right)\right|=\sum_{k=0}^{q_{n}-1} \int_{A^{c} \cap V_{n}} D T^{k}(x) d x \leq \frac{e^{v}\left|A^{c} \cap V_{n}\right|}{\left|V_{n}\right|} \sum_{k=0}^{q_{n}-1}\left|T^{k}\left(V_{n}\right)\right| \leq 2 e^{v} \epsilon .
$$

Since $\epsilon$ was arbitrary, $\left|A^{c}\right|=0$. The theorem is proved.

Lemma 2.5. Let $T_{1}$ and $T_{2}$ are $\mathfrak{B}$-homeomorphisms with identical irrational rotation number. Then the conjugation map $h$ between $T_{1}$ and $T_{2}$ is either absolutely continuous or singular function.

Proof. Consider two $\mathfrak{B}$-homeomorphisms $T_{1}$ and $T_{2}$ of the circle $S^{1}$ with identical irrational rotation number $\rho$. Let $\varphi_{1}$ and $\varphi_{2}$ be maps conjugating $T_{1}$ and $T_{2}$ with the rigid rotation $T_{\rho}$, that is, $\varphi_{1} \circ T_{1}=T_{\rho} \circ \varphi_{1}$ and $\varphi_{2} \circ T_{2}=T_{\rho} \circ \varphi_{2}$. It is easy to check that the map $h=\varphi_{2}^{-1} \circ \varphi_{1}$ conjugates $T_{1}$ and $T_{2}$, that is

$$
h \circ T_{1}=T_{2} \circ h .
$$

We know that conjugation function $h$ is strictly increasing function on $S^{1}$. Then $D h$ exists almost everywhere on $S^{1}$. Denote by $A=\left\{x: x \in S^{1}, D h(x)>0\right\}$. It is clear that the set $A$ is mod 0 invariant with respect to $T_{1}$. Since the class $\mathfrak{B}$-homeomorphism is ergodic with respect to the Lebesgue measure. Hence, the Lebesgue measure of set $A$ is either null or full. If Lebesque measure of $A$ is null then $h$ is a singular function, if it is full then $h$ is an absolutely continuous function.

Remark 2.6. Let $T_{1}$ and $T_{2}$ be $\mathfrak{B}$-homeomorphisms with identical irrational rotation number. Then conjugation map $h^{-1}$ between $T_{2}$ and $T_{1}$ is either absolutely continuous or singular.

\section{Martingales and Martingale Convergence Theorem}

Our objective in this section is to develop the fundamentals of the theory of martingales and prepare for the main results and applications that will be presented in the subsequent sections.

Definition 3.1. Let $(X, \mathfrak{F})$ be a measurable space. A sequence $\left(\mathfrak{F}_{m}\right)$ of $\sigma$-algebras on $X$ is said to be a filtration in $\mathfrak{F}$, if

$$
\mathfrak{F}_{1} \subseteq \mathfrak{F}_{2} \subseteq \cdots \subseteq \mathfrak{F}
$$

Statement 3.2. The sequence of algebras generated by dynamical partitions, which is also denoted by $\left(\mathbf{P}_{m}\right)$ (by abuse of notation) is a filtration in $\boldsymbol{B}$, where $\boldsymbol{B}$ is a Borel $\sigma$-algebra on $S^{1}$.

Definition 3.3. Let $\left(\mathcal{R}_{m}\right)$ be a sequence of random variables on a measurable space $(X, \mathfrak{F})$ and $\left(\mathfrak{F}_{m}\right)$ a filtration in $\mathfrak{F}$. We say that $\left(\mathcal{R}_{m}\right)$ is adapted to $\left(\mathfrak{F}_{m}\right)$ if, for each positive integer $m, \mathcal{R}_{m}$ is $\mathfrak{F}_{m}$-measurable. 
Denote by $E(\mathcal{R} \mid \mathfrak{F})$ conditional expectation of random variables $R$ with respect to partition $\mathfrak{F}$.

Definition 3.4. Let $\left(\mathcal{R}_{m}\right)$ be a sequence of random variables on a probability space $(X, \mathfrak{F}, \mathbf{P})$ and $\left(\mathfrak{F}_{m}\right)$ a filtration in $\mathfrak{F}$. The sequence $\left(\mathcal{R}_{m}\right)$ is said to be a martingale with respect to $\left(\mathfrak{F}_{m}\right)$ if, for every positive integer $m$,

(i) $\left(\mathcal{R}_{m}\right)$ is integrable;

(ii) $\left(\mathcal{R}_{m}\right)$ is adapted to $\left(\mathfrak{F}_{m}\right)$;

(iii) $E\left(\mathcal{R}_{m+1} \mid \mathfrak{F}_{m}\right)=\mathcal{R}_{m}$.

Lemma 3.5 (see [12]). Let $\left(\mathcal{R}_{m}\right)$ be a sequence of random variables on a probability space $(X, \mathfrak{F}, \mathbf{P})$. If $\sup _{m} E\left(\left|\mathcal{R}_{m}\right|^{p}\right)<\infty$ for some $p>1$ and $\left(\mathcal{R}_{m}\right)$ is a martingale, then there exists an integrable $\mathcal{R} \in L_{1}(X, \mathfrak{F})$ such that

$$
\lim _{m \rightarrow \infty} \mathcal{R}_{m}=\mathcal{R}(\text { a.e } \mathbf{P}), \quad \mathcal{R}_{m} \longrightarrow \mathcal{R} \text { in } L_{1}-\text { norm } .
$$

Suppose $f$ is a homeomorphism (not necessary to be $\mathfrak{B}$-homeomorphism) of the circle $S^{1}$. Using the homeomorphism $f$ and sequence of dynamical partitions $\left(\mathbf{P}_{m}\right)$, we define the sequence of random variables on the circle which is generating a martingales. For any $m \geq 1$, we set

$$
\mathcal{R}_{m}(x)=\frac{\left|f\left(\Delta^{m}\right)\right|}{\left|\Delta^{m}\right|}, \quad \text { if } x \in \Delta^{m}, \Delta^{m} \in \mathbf{P}_{m}
$$

Lemma 3.6. The sequence $\left(\boldsymbol{R}_{m}\right)$ of random variables is a martingale with respect to $\left(\mathbf{P}_{m}\right)$.

Proof. To prove the martingale, it suffices to check $E\left(\mathcal{R}_{m+1} \mid \mathbf{P}_{m}\right)=\mathcal{R}_{m}$, for any $m \geq 1$, because the sequence of random variables $\left(\mathcal{R}_{m}\right)$ is sequence of step functions, so the sequence of step functions is integrable and adapted to $\left(\mathbf{P}_{m}\right)$. Denote by $\chi_{I}$ indicator function of interval $I$. Using definition of conditional expectation of random variables $\left(\mathcal{R}_{m}\right)$ with respect to partition $\left(\mathbf{P}_{m}\right)$, we get

$$
E\left(\mathcal{R}_{m+1} \mid \mathbf{P}_{m}\right)=\sum_{i=0}^{q_{m-1}-1} E\left(\mathcal{R}_{m+1} \mid \Delta_{i}^{m}\right) \chi_{\Delta_{i}^{m}}+\sum_{i=0}^{q_{m}-1} E\left(\mathcal{R}_{m+1} \mid \Delta_{i}^{m-1}\right) \chi_{\Delta_{i}^{m-1}} .
$$

Now, we calculate each sum of (3.4) separately. Note, that each interval of $\mathbf{P}_{m}$ order $m$ is member of $\mathbf{P}_{m+1}$ and each interval $\Delta_{i}^{m-1} \in \mathbf{P}_{m}, 0 \leq i<q_{m}$, is partitioned into $k_{m+1}+1$ intervals belonging to $\mathbf{P}_{m+1}$ such that

$$
\Delta_{i}^{m-1}=\Delta_{i}^{m+1} \cup \bigcup_{s=0}^{k_{m+1}-1} \Delta_{i+q_{m-1}+s q_{m}}^{m}
$$


Using this, we get

$$
\begin{aligned}
& E\left(\mathcal{R}_{m+1} \mid \Delta_{i}^{m}\right)=\frac{1}{\left|\Delta_{i}^{m}\right|} \int_{\Delta_{i}^{m}} \mathcal{R}_{m+1}(x) \ell(d x)=\frac{1}{\left|\Delta_{i}^{m}\right|} \int_{\Delta_{i}^{m}} \mathcal{R}_{m}(x) \ell(d x) \\
& E\left(\mathcal{R}_{m+1} \mid \Delta_{i}^{m-1}\right)= \frac{1}{\left|\Delta_{i}^{m-1}\right|} \int_{\Delta_{i}^{m-1}} \mathcal{R}_{m+1}(x) \ell(d x)=\frac{1}{\left|\Delta_{i}^{m-1}\right|}\left[\int_{\Delta_{i}^{m+1}} \mathcal{R}_{m+1}(x) \ell(d x)\right] \\
&+\frac{1}{\left|\Delta_{i}^{m-1}\right|}\left[\sum_{s=0}^{k_{m+1}-1} \int_{\Delta_{i+q_{m-1}+s q m}^{m}} \mathcal{R}_{m+1}(x) \ell(d x)\right] \\
&= \frac{1}{\left|\Delta_{i}^{m-1}\right|} \int_{\Delta_{i}^{m-1}} \mathcal{R}_{m}(x) \ell(d x) .
\end{aligned}
$$

Finally, summing (3.4), (3.6), and (3.7), we get

$$
E\left(\mathcal{R}_{m+1} \mid \mathbf{P}_{m}\right)=\sum_{i=0}^{q_{m-1}-1} \mathcal{R}_{m}(x) \chi_{\Delta_{i}^{m}}+\sum_{i=0}^{q_{m}-1} \mathcal{R}_{m}(x) \chi_{\Delta_{i}^{m-1}}=\mathcal{R}_{m}
$$

The following inequality (sometimes called "parallelogram inequality") is useful for estimating fractions, and we will use it in the proof of the next statement.

Lemma 3.7. Given $a, b, c, d>0$, the following inequalities hold

$$
\min \left\{\frac{a}{b}, \frac{c}{d}\right\} \leq \frac{a+c}{b+d} \leq \max \left\{\frac{a}{b}, \frac{c}{d}\right\}
$$

Proof. Consider points $A=(a, b), B=(c, d)$, and $C=(a+c, b+d)$ on the plan $x O y$. The slope of the ray $O C$ lies between slops of rays $O A$ and $O B$.

\section{Proof of Main Theorems}

Let $h$ be the conjugation homeomorphism between $T_{1}$ and $T_{2}$, that is, $h \circ T_{1}=T_{2} \circ h$. Without loss of generality, we assume $h(0)=0$. Consider dynamical partition $\mathbf{P}_{m}\left(T_{1}\right)$. Define sequence of random variables $\left(\mathcal{R}_{m}\right)$ on the $S^{1}$ by this formula

$$
\mathcal{R}_{m}(x)=\frac{\left|h\left(\Delta^{m}\right)\right|}{\left|\Delta^{m}\right|}, \quad \text { if } x \in \Delta^{m}, \Delta^{m} \in \mathbf{P}_{m}\left(T_{1}\right)
$$

Denote by $\Theta_{m}(x)=\mathcal{R}_{m}(x)-\mathcal{R}_{m-1}(x), m \geq 1$ and $\mathcal{R}_{0}(x): \equiv 0, x \in S^{1}$. 
Statement 4.1. Let the sequence $\left(\tau_{m}\right)$ be defined in Theorem 1.3. Then there exists a universal constant $C_{4}=C_{4}(T)>0$ such that for all $m \geq 1$, the following inequality holds

$$
\left|\Theta_{m}(x)\right| \leq C_{4} \tau_{m}\left|\mathcal{R}_{m-1}(x)\right|, \quad x \in S^{1} .
$$

Proof. It is clear that

$$
\left|\Theta_{m}(x)\right|=\left|\mathcal{R}_{m-1}(x)\right|\left|\frac{\left|\mathcal{R}_{m}(x)\right|}{\left|\mathcal{R}_{m-1}(x)\right|}-1\right| .
$$

Now, we estimate $\mathcal{R}_{m}(x) / \mathcal{R}_{m-1}(x)$. Denote by $\mathcal{R}_{m}\left(\Delta^{m}\right):=\mathcal{R}_{m}(x), x \in \Delta^{m}$ and $\Delta^{m} \in \mathbf{P}_{m}\left(T_{1}\right)$. Thus, we have

$$
\left|\Delta^{m-1}\right| \mathcal{R}_{m-1}\left(\Delta^{m-1}\right)=\sum_{s=0}^{k_{m}}\left|\Delta^{m}(s)\right| \mathcal{R}_{m}\left(\Delta^{m}(s)\right),
$$

where $\Delta^{m}(s) \subset \Delta^{m-1}$. Using Lemma 3.7, we get

$$
\min \mathcal{R}_{m}\left(\Delta^{m}(s)\right) \leq \mathcal{R}_{m-1}\left(\Delta^{m-1}\right) \leq \max \mathcal{R}_{m}\left(\Delta^{m}(s)\right)
$$

It is clear that for any $0 \leq s \leq k_{m}$ holds

$$
\frac{\min \mathcal{R}_{m}\left(\Delta^{m}(s)\right)}{\max \mathcal{R}_{m}\left(\Delta^{m}(s)\right)} \leq \frac{\mathcal{R}_{m-1}\left(\Delta^{m-1}\right)}{\mathcal{R}_{m}\left(\Delta^{m}(s)\right)} \leq \frac{\max \mathcal{R}_{m}\left(\Delta^{m}(s)\right)}{\min \mathcal{R}_{m}\left(\Delta^{m}(s)\right)}
$$

Since, each pair of adjacent intervals of $\mathbf{P}_{m}\left(T_{1}\right)$ are $C_{2}$-comparable. By the assumption of Theorem 1.3, we get

$$
\left|\frac{\mathcal{R}_{m}\left(\Delta^{m}(s+1)\right)}{\mathcal{R}_{m}\left(\Delta^{m}(s)\right)}-1\right| \leq C_{2} \tau_{m}
$$

Hence, the rotation number $\rho=\left[k_{1}, k_{2}, \ldots, k_{m}, \ldots\right]$ is of bounded type, and an easy trick gives us

$$
\frac{\max \mathcal{R}_{m}\left(\Delta^{m}(s)\right)}{\min \mathcal{R}_{m}\left(\Delta^{m}(s)\right)} \leq\left(1+C_{2} \tau_{m}\right)^{K} \leq 1+C_{4} \tau_{m}
$$

where $K=\sup \left(k_{m}\right)$ and $C_{4}=K C_{2}$. A similar lower bound holds true for $\min \mathcal{R}_{m}\left(\Delta^{m}(s)\right)$ : $\max \mathcal{R}_{m}\left(\Delta^{m}(s)\right)$. Therefore, we have

$$
1-C_{4} \tau_{m} \leq \frac{\mathcal{R}_{m-1}\left(\Delta^{m-1}\right)}{\mathcal{R}_{m}\left(\Delta^{m}(s)\right)} \leq 1+C_{4} \tau_{m}
$$

for all $0 \leq s \leq k_{m}$. 
Proof of Theorem 1.3. For the proof of Theorem 1.3, we use the above reasonings. By Lemma 3.6, the sequence $\left(\boldsymbol{R}_{m}\right)$ of random variables is a martingale with respect to $\left(\mathbf{P}_{m}\right)$. We want to show that $\mathcal{R}_{m}$ converges to $D h$ in the norm $L_{1}\left(S^{1}, d \ell\right)$ when $m \rightarrow \infty$. By direct calculation, it is easy to see that $\Theta_{m}(x)$ and $\mathcal{R}_{m-1}(x)$ is orthogonal, that is

$$
\int_{S^{1}} \Theta_{m}(x) \mathcal{R}_{m-1}(x) d x=0 .
$$

Using the assertion of Statement 4.1, we get

$$
\left\|\mathcal{R}_{m}\right\|_{L_{2}}^{2} \leq\left\|\mathcal{R}_{m-1}\right\|_{L_{2}}^{2}+\left\|\Theta_{m}\right\|_{L_{2}}^{2} \leq\left(1+C_{4} \tau_{m}^{2}\right)\left\|\mathcal{R}_{m-1}\right\|_{L_{2}}^{2} .
$$

Iterating the last relation, we have $\left\|\mathcal{R}_{m}\right\|_{L_{2}}^{2} \leq \prod_{j=1}^{m}\left(1+C_{4} \tau_{j}^{2}\right)$. So far as the series $\sum_{j=1}^{\infty} \tau_{j}^{2}$ converges. From this implies that the sequence of random variables $\left(\mathcal{R}_{m}\right)$ is bounded in $L_{2}$ norm. By Lemma 3.5, the sequence of random variables $\left(R_{m}\right)$ converges to some function $R$ in $L_{1}$ norm. We prove that sequence of random variables $\left(\mathcal{R}_{m}\right)$ converges to the $D h$. Indeed, denote by $\alpha_{m}$ and $\beta_{m}$ end points of interval $\Delta^{m}$ of dynamical partition $\mathbf{P}_{m}\left(T_{1}\right)$. By definition of $\mathcal{R}_{m}$, we have

$$
\left|h(x)-\int_{0}^{x} \mathcal{R}_{m}(x) d x\right| \leq\left|h(x)-h\left(\alpha_{m}\right)\right|+\frac{\left|h\left(\Delta^{m}\right)\right|}{\left|\Delta^{m}\right|}\left|x-\alpha_{m}\right| \leq 2\left|h\left(\Delta^{m}\right)\right| .
$$

Moreover, using last inequality, we obtain

$$
\left|h(x)-\int_{0}^{x} \mathcal{R}(x) d x\right| \leq\left|h(x)-\int_{0}^{x} \mathcal{R}_{m}(x) d x\right|+\int_{0}^{x}\left|\mathcal{R}(x)-\mathcal{R}_{m}(x)\right| d x \leq 2\left|h\left(\Delta^{m}\right)\right|+\left\|\mathcal{R}_{m}-\mathcal{R}\right\|_{L_{1}} .
$$

From this taking the limit when $m \rightarrow \infty$, we get $h(x)=\int_{0}^{x} \mathcal{R}(x) d x$. Since, $\mathcal{R} \in L_{1}\left(S^{1}, d \ell\right)$, then $h$ is absolutely continuous function and $D h(x)=R(x)$ almost everywhere on $S^{1}$. Thus, Theorem 1.3 is completely proved.

Statement 4.2. For all $\psi \in L_{1}\left(S^{1}, d \ell\right)$ hold this equality

$$
\lim _{m \rightarrow \infty}\left\|\psi \circ T_{1}^{q_{m}}-\psi\right\|_{L_{1}}=0
$$

Proof. It is a well-known fact that the class $C([a, b])$ of continuous functions on $[a, b]$ is dense (in $\left.\|\cdot\|_{L_{1}}\right)$ in $L_{1}\left([a, b], d \ell\right.$ ) (see [13]). From this fact it implies that if $\psi \in L_{1}\left(S^{1}, d \ell\right)$, then for any $\epsilon>0$ there exists a continuous function $\psi_{\epsilon} \in C\left(S^{1}\right)$ and $\phi_{\epsilon} \in L_{1}\left(S^{1}, d \ell\right)$ such that $\psi=\psi_{\epsilon}+\phi_{\epsilon}$ and $\left\|\phi_{\epsilon}\right\|_{L_{1}} \leq \epsilon$. Using this and Denjoy estimate, we obtain

$$
\begin{aligned}
\left\|\psi \circ T_{1}^{q_{m}}-\psi\right\|_{L_{1}} & \leq\left\|\psi_{\epsilon} \circ T_{1}^{q_{m}}-\psi_{\epsilon}\right\|_{L_{1}}+\left(\sup \left|D T_{1}^{q_{m}}\right|^{-1}+1\right)\left\|\phi_{\epsilon}\right\|_{L_{1}} \\
& \leq\left\|\psi_{\epsilon} \circ T_{1}^{q_{m}}-\psi_{\epsilon}\right\|_{L_{1}}+\left(1+e^{v}\right)\left\|\phi_{\epsilon}\right\|_{L_{1}} .
\end{aligned}
$$


As $\psi_{\epsilon}$ is uniformly continuous on $S^{1}$ and by exponential refinement $T_{1}^{q_{m}}(x)$ uniformly tends to $x$, there exists a positive integer $m_{0}=m_{0}(\epsilon)$ such that for all $m \geq m_{0}$, the $\left\|\psi_{\epsilon} \circ T_{1}^{q_{m}}-\psi_{\epsilon}\right\|_{L_{1}} \leq \epsilon$. Therefore, $\left\|\psi \circ T_{1}^{q_{m}}-\psi\right\|_{L_{1}} \leq\left(2+e^{v}\right) \epsilon$. Since $\epsilon>0$ was arbitrary and sufficiently small.

Proof of Theorem 1.4. Assume that conjugation map $h$ is absolutely continuous, then $D h \in$ $L_{1}\left(S^{1}, d \ell\right)$ and $D h>0$ almost everywhere. For all positive integers $n$, the function $D h$ satisfies

$$
\left(D h \circ T_{1}^{q_{n}}\right) D T_{1}^{q_{n}}=D\left(T_{2}^{q_{n}} \circ h\right) D h \text { a.e. }
$$

Taking the logarithm, we obtain

$$
\log \left(D h \circ T_{1}^{q_{n}}\right)-\log D h=\log D\left(T_{2}^{q_{n}} \circ h\right)-\log D T_{1}^{q_{n}} .
$$

Denote by $\psi=\log D h$, it is clear that $\psi \in L_{1}\left(S^{1}, d \ell\right)$. Suppose, by contradiction, that there exists $\delta>0$, such that the Lebesgue measure of the set $S_{\delta}^{n}=\left\{x: \mid \log D T_{2}^{q_{n}}(h(x))-\right.$ $\left.\log D T_{1}^{q_{n}}(x) \mid \geq \delta\right\}$ does not converge to 0 when $n$ goes to infinity. Hence, for all positive integer $n$ :

$$
\int_{S^{1}}\left|\psi\left(T_{1}^{q_{n}}(x)\right)-\psi(x)\right| d x \geq \int_{S_{\delta}^{n}}\left|\psi\left(T_{1}^{q_{n}}(x)\right)-\psi(x)\right| d x \geq \delta\left|S_{\delta}^{n}\right|
$$

But $\left|S_{\delta}^{n}\right|$ does not tend to 0 when $n$ goes to $+\infty$. Hence $\int_{S^{1}}\left|\psi\left(T_{1}^{q_{n}}(x)\right)-\psi(x)\right| d x$ does not tend to 0 when $n$ goes to $+\infty$, this contradicts Statement 4.2 and ends the proof of Theorem 1.4.

\section{Acknowledgments}

The authors are grateful to A. A. Dzhalilov for useful discussions and grateful to Universiti Kebangsaan Malaysia for providing financial support via the Grants UKM-MI-OUP-2011 and UKM-DIP-2012-31. They also thank the referee for comments that have improved the presentation of this paper.

\section{References}

[1] I. P. Cornfeld, S. V. Fomin, and Y. G. Sinai, Ergodic Theory, Springer, Berlin, Germany, 1982.

[2] A. Y. Khinchin, Continued Fractions, University of Chicago Press, 1964.

[3] K. M. Khanin and E. B. Vul, "Circle homeomorphisms with weak discontinuities," in Dynamical Systems and Statistical Mechanics, vol. 3 of Advances in Soviet Mathematics, pp. 57-98, 1991.

[4] Y. Katznelson and D. Ornstein, "The differentiability of the conjugation of certain diffeomorphisms of the circle," Ergodic Theory and Dynamical Systems, vol. 9, no. 4, pp. 643-680, 1989.

[5] K. M. Khanin and Y. G. Sinai, "Smoothness of conjugacies of diffeomorphisms of the circle with rotations," Russian Mathematical Surveys, vol. 44, no. 1, pp. 69-99, 1989, translation of Uspekhi Matematicheskikh Nauk, vol. 44, pp. 57-82, 1989.

[6] K. Khanin and A. Y. Teplinskii, "Herman's theory revisited," Inventiones Mathematicae, vol. 178, no. 2, pp. 333-344, 2009.

[7] A. Y. Teplinskii and K. M. Khanin, "Rigidity for circle diffeomorphisms with singularities," Russian Mathematical Surveys, vol. 59, no. 2, pp. 329-353, 2004, translation of Uspekhi Matematicheskikh Nauk, vol. 59, no. 2, pp. 137-160, 2004. 
[8] A. A. Dzhalilov, H. Akin, and S. Temir, "Conjugations between circle maps with a single break point," Journal of Mathematical Analysis and Applications, vol. 366, no. 1, pp. 1-10, 2010.

[9] Y. Katznelson and D. Ornstein, "The absolute continuity of the conjugation of certain diffeomorphisms of the circle," Ergodic Theory and Dynamical Systems, vol. 9, no. 4, pp. 681-690, 1989.

[10] A. A. Dzhalilov and I. Liousse, "Circle homeomorphisms with two break points," Nonlinearity, vol. 19, no. 8, pp. 1951-1968, 2006.

[11] A. A. Dzhalilov, D. Mayer, and U. A. Safarov, "Piecewise-smooth circle homeomorphisms with several break points," Izvestiya, vol. 76, no. 1, pp. 94-113, 2012, translation of Izvestiya Rossiiskoi Akademii Nauk, vol. 76, no. 1, pp. 101-120, 2012.

[12] A. N. Shiryayev, Probability, New York, NY, USA, 1984.

[13] M. Reed and B. Simon, Methods of Modern Mathematical Physics, San Diego, Calif, USA, 1980. 


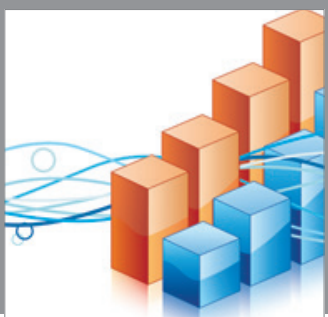

Advances in

Operations Research

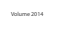

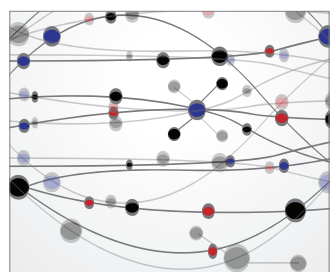

\section{The Scientific} World Journal
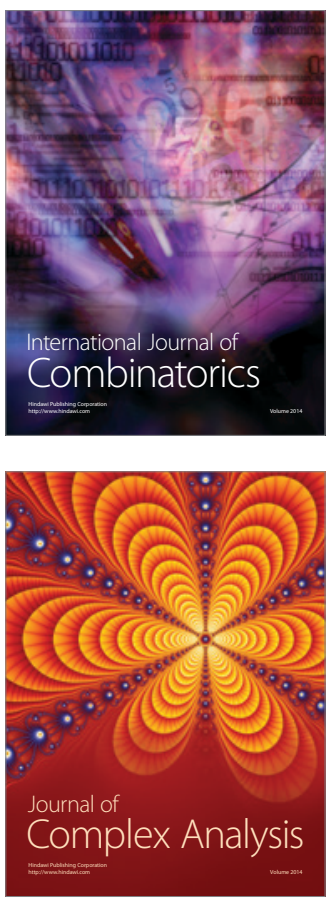

International Journal of

Mathematics and

Mathematical

Sciences
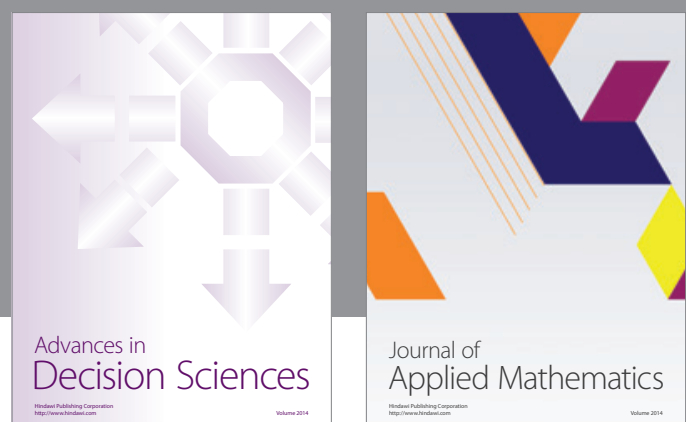

Journal of

Applied Mathematics
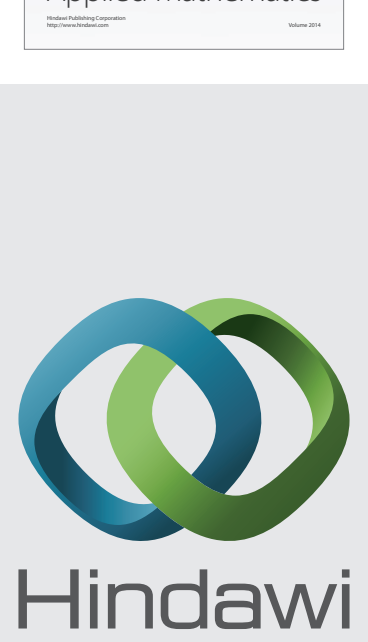

Submit your manuscripts at http://www.hindawi.com
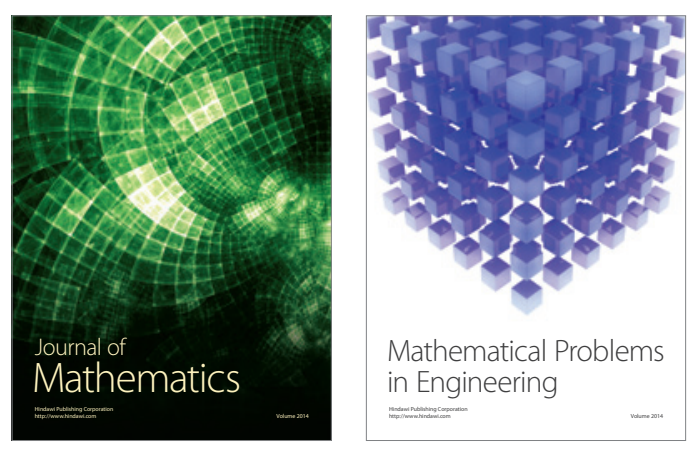

Mathematical Problems in Engineering
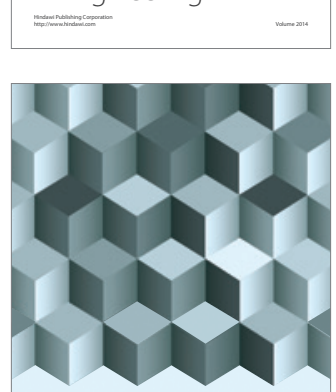

Journal of

Function Spaces
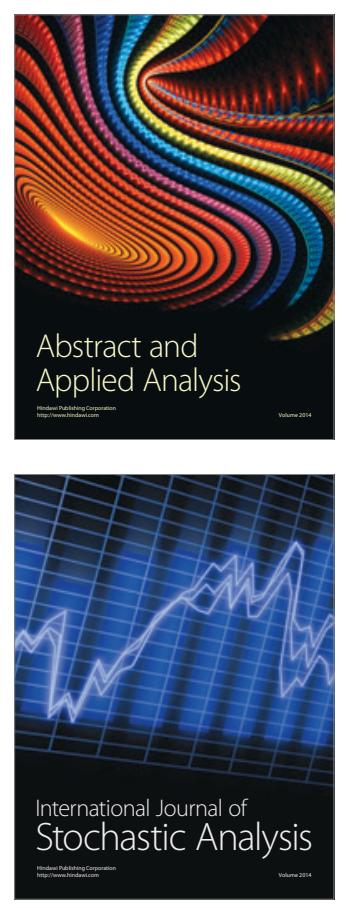

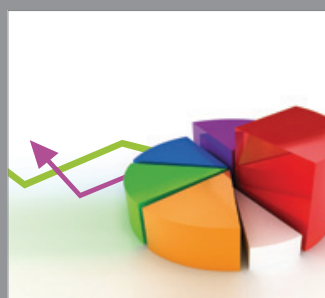

ournal of

Probability and Statistics

Promensencen
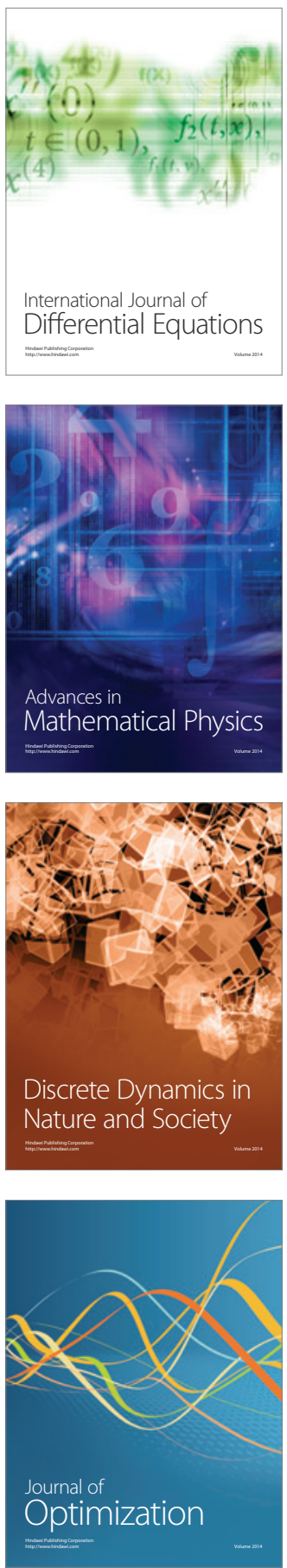